\title{
Covalent attachment of biotinylated molecular beacons via thiol-ene coupling. A study on conformational changes upon hybridization and streptavidin binding
}

\author{
Daniel González-Lucas, ${ }^{1}$ María-José Bañuls, ${ }^{1}$ Jaime García-Rupérez, ${ }^{2}$ Ángel Maquieira $* 1$
}

Keywords: click chemistry, dual polarization interferometry, biosensing, Silicon on insulator, hybridization

\begin{abstract}
The authors describe a method for the detection of DNA by using immobilized molecular beacons (MBs) on the surface of silicon, with a view on possible application in biosensing. MB hybridization and protein recognition was interrogated on silicon-on-insulator (SOI) surfaces by using fluorescently tagged probes. In order to better understand the conformational changes that occur to MBs upon hybridization, the process was studied by using dual polarization interferometry (DPI). A model system was developed that matches thickness, mass, and density parameters. The results experimentally demonstrates for the first time that hybridization promotes the displacement of a protein away from the surface. This finding may be further exploited in techniques such as photonic sensors, thereby paving the way to the design of more sensitive biosensors based on the use of MBs.
\end{abstract}

\section{INTRODUCTION}

DNA sensors have garnered wide interest as potential companion diagnostics in personalized medicine, early cancer detection, forensic investigation, assessment of medical treatment and other applications.[1-4] Sensitive detection of specific nucleic acid sequences on the basis of the hybridization reaction can be improved by target or signal amplification strategies. A series of novel principles, methods and techniques based on molecular beacon probes have been developed for bioanalysis and biomedical research.[5-7]

Molecular beacons (MBs) are a class of DNA probes which are single stranded oligonucleotides that possess a stem-loop structure and are an excellent example of biomolecular analysis probe that appears to be very promising for genomic studies.[6,7] The thermodynamic stability of the hairpin structure, its highly efficient intrinsic signal switching and the possibility of using a wide variety of markers, make MBs exceptional biomolecular analysis probes with excellent sensitivity, selectivity and real-time detection capability.

When a MB hybridizes with its complementary strand, it undergoes a spontaneous conformational reorganization with the opening of the stem. This conformational reorganization has been exploited in the literature in different ways.[6,7] Adding a fluorophore and a quencher is the most common form as fluorescence appearance is directly associated with the formation of the double strand in solution (opened form of the MB and the complementary strand). This makes the MB a highly sensitive and selective DNA probe to report label-free targets.

However, few studies exploit the MB hairpin conformation on surfaces for the detection of DNA by hybridization measuring fluorescence. $[8,9]$ Some studies were reported where MBs incorporating a nanoparticle (NP) were exploited for biosensing in surfaces as the hybridization promotes the displacement of the NP away from the surface giving rise to the signal and very high LODs.[10] In all cases, the opening of the MB have been obviated, assuming that MBs behave on a similar manner in solution as they do when immobilized in solid supports. When hybridization takes place in solid supports there are many other factors that needs to be considered as it was

${ }^{1}$ IDM, Instituto Interuniversitario de Investigación de Reconocimiento Molecular y Desarrollo Tecnológico, Departamento de Química, Universitat Politècnica de València, Universitat de València, Valencia, Spain. E-mail address: amaquieira@qim.upv.es (Á. Maquieira).Tel.: +34 96 3873415; fax: +34 963873415.

${ }^{2}$ Nanophotonics Technology Center, Universitat Politècnica de València, Valencia, Spain

Electronic Supplementary Material: The online version of this article (DOI: 10.1039/x0xx00000x) contains supplementary material, which is available to authorized users. 
previously demonstrated by our group.[11] Therefore to the best of our knowledge this is the first report that experimentally demonstrates the opening of the hairpin structure for the final successful application of MBs in biosensing.

The site-specific immobilization of MBs and its hybridization with a complementary target is therefore opening wide interest.[9,12,8,10,13] As a result of its orthogonality and the ability to selectively pattern surfaces by simple exposure to UV light, thiol-ene chemistry appears to be optimal to covalently attach MBs to surfaces in a biocompatible manner.[11,14] Thus, in this work, we use for the first time thiol-ene chemistry (TEC) to attach MBs to a silicon-based surface and study its hybridization ability after immobilization.

For the application of MBs in most techniques, different reporters need to be attached to them. The most common way to incorporate reporters are coupling reactions through $\mathrm{NH}_{2}$ or $\mathrm{COOH}$ modifications in the MBs.[15,10] In this work, we propose the idea of adding an extra biotin arm close to one of the ends of the MB. MBs incorporating biotin can open up a wide range of applications of MBs in biosensing techniques. Several streptavidin-linked reporters, such as proteins or nanoparticles, can be directly attached to MBs using the very robust[16] biotinstreptavidin recognition. Therefore, the conformational change promoted by hybridization changes the disposition of the streptavidin and the reporter, being this fact of special interest in the modulation of the signal in biosensing.

Dual polarization Interferometry (DPI) is currently one of the most powerful analytical tools in heterogeneous format to record real-time data of conformational dynamics, which is efficiently employed in different applications, such as bionanotechnology, surface science, and crystallography or drug discovery. ${ }^{[17]}$ The information it delivers is the interaction of two polarization modes of the propagating light with a molecular film at the top of the waveguide with which it interacts through the evanescent field. DPI therefore allows refractive index (RI), layer thickness, mass and density parameters of the monolayer to be measured almost simultaneously as it measures two different interference fringe patterns at the same time.

Chips for DPI measurements are made of silicon oxynitride, which makes it possible to employ the same thiol-ene chemistry (TEC) set up for fluorescence microarrays. Besides, each DPI chip contains two channels exposed to flow, available for the measurement and a third one as reference channel, which is not in contact with the sample flowing over the chip. By studying the hybridization of MB incorporating a protein on DPI, we successfully proved that the opening of the hairpin structure takes place on the surface of the chip, promoting the displacement of the protein away from the surface without significant loss in performance upon hybridization. Therefore, for the study of suitability of the MB structure in biosensors, a model of the MB hybridization process incorporating a high density protein is proposed, with measured distances, MB structures and positions related to the surface. These studies will be of great interest for the future development of novel label-free biosensors that exploits the hairpin conformational changes of MBs upon hybridization.

\section{MATERIALS AND METHODS}

\subsection{Reagents}

All chemicals were purchased from Sigma-Aldrich (Spain, www.sigmaaldrich.com) and used without further purification. All labelled and functionalized oligonucleotides were purchased from Eurofins Genomic (Europe, www.eurofinsgenomics.eu) and used without any further modification, the sequences and functionalizations are included in table 1 . All other common chemicals were analytical grade and Milli-Q purified water was employed in all stages $\left(18.2 \mathrm{M} \Omega \cdot \mathrm{cm}^{-1}\right)$. Phosphate buffered saline (PBS, $10 \mathrm{mM}$ phosphate, $137 \mathrm{mM} \mathrm{NaCl}, 2.7 \mathrm{mM} \mathrm{KCl}, \mathrm{pH} 7.4$, $0.5 \%$ Tween 20 was added to obtain PBST) and sodium saline citrate solutions (SSC, $750 \mathrm{mM} \mathrm{NaCl}, 75 \mathrm{mM}$ sodium citrate, $\mathrm{pH} 7$ ) were used as a carrier or medium in all assays. For the DPI analysis, buffered solutions were previously filtered through a $0.45 \mu \mathrm{m}$ PVDF filter and degassed by sonication under vacuum prior to be employed. SOI chips $(2 \times 1 \mathrm{~cm})$ were obtained from wafers from SIEGERT WAFER GmbH (Aachen, Germany, www.siegertwafer.com/). Microarray printing was carried out with a low-volume noncontact dispensing system from Biodot (Irvine, CA, USA, www.biodot.com), model AD1500. The polymerizations and photografting were carried out on a surface cleaner $\left(254 \mathrm{~nm}, 50 \mathrm{~mW} \cdot \mathrm{cm}^{-2}\right)$ not connected to oxygen.

\subsection{Experimental techniques}


The fluorescence signal of microarrays was registered with a homemade surface fluorescence reader (SFR) having a high sensitivity charge coupled device camera Retiga EXi from Qimaging, Inc. (Burnaby, Canada), with light emitting diodes Toshiba TLOH157P as light source.[18] For microarray image processing and subsequent quantification, GenePix Pro 4.0 software from Molecular Devices, Inc. (Sunnyvale, CA, USA), was employed. WCA measurements were performed with a Dino-lite digital microscope (model AM-413ZT from Dino-Lite Europe, http://www.dino-lite.com) and processed with Dinocapture 1.5.0 software.

DPI experiments were undertaken at $20^{\circ} \mathrm{C}$ with an Analight Bio200 Dual Polarization Interferometer (Biolin Scientific, Stockholm, Sweden, http://www.biolinscientific.com) using silicon oxynitride chips (FB 100, Biolin Scientific, Stockholm, Sweden, http://www.biolinscientific.com). The sensor surface has two fluidic interfaces $(2 \mu \mathrm{L}$ dead volume each, named channel 1 and channel 3) with an additional waveguide reference area (channel 2) having a dielectric cover with constant RI. Carrier and other solutions were flowed using a double-channel precision syringe pump (Harvard Apparatus PHD 2000 Infusion, Kent, UK, http://www.harvardapparatus.com) and injections were carried out with a dual injection valve. Analight DAQ and Analight Explorer (Biolin, Sweden) software packages were used for system managing/data acquisition and data treatment, respectively.

Table 1. Oligonucleotide sequences used

\begin{tabular}{llll}
\hline Name & $5^{\prime}$ end & $3^{\prime}$ end & sequence $\left(5^{\prime}-3^{\prime}\right)$ \\
\hline MB 1 & Cy5 & SH & CCCGATZTGACCAGCTAGCATTCGGG \\
\hline MB 2 & - & SH & CCCGATZTGACCAGCTAGCATTCGGG \\
\hline MB 3 & & SH & ATTCACTCAACATCAGTCTGGTAAGCTAGTGAAT \\
\hline MB 4 & SH & - & ATCGACACCCCTATCACGATTAGCATTAAGZTCGAT \\
\hline Target 1 & Cy5 & - & AATGCTAGCTGGTCAATCGGG \\
\hline Target 2 & Cy5 & - & UAGCUUAUCAGACUGAUGUUGA \\
\hline Target 3 & Cy5 & - & AUCGACUUAAUGCUAAUCGUGAUAGGGGUGUCGAU \\
\hline Probe 1 & SH & - & $(\text { T })_{15}$-CCCGATTGACCAGCTAGCATT \\
\hline Probe 2 & Cy5 & SH & $(\text { T })_{15}$-CCCGATTGACCAGCTAGCATT \\
\hline *Z denotes biotin functionalization
\end{tabular}

\subsection{Experimental procedures}

2.3.1. Functionalization of chips. Commercial silicon-on-insulator (SOI) chips were treated with a UV ozone surface cleaner for $10 \mathrm{~min}$. Then the chips were immersed in a $2 \%$ solution of vinyltriethoxysilane in dry toluene. The chips were incubated for $2 \mathrm{~h}$ in the solution, rinsed with acetone and dried with air. The dried chips were finally cured in an oven at $80{ }^{\circ} \mathrm{C}$ for $30 \mathrm{~min}$, washed with acetone and stored protected from light. Water contact angle of $73.4^{\circ}$ was obtained for the vinyl-functionalized chips by measuring the contact angle of a $4 \mu \mathrm{L}$ milliQ water drop as the mean of 10 measurements in different chips. Chips functionalized with this procedure were stable for at least 4 weeks without any observed decrease in performance.

Silicon oxinitride chips (DPI) were washed with EtOH and activated with a UV ozone cleaner for $10 \mathrm{~min}$. Then the chips were functionalized with vinyltriethoxysilane by chemical vapor deposition overnight at $400 \mathrm{mmHg}$. The functionalized chips were carefully washed with isopropanol and dried with air (WCA: $80.1^{\circ}$ ).

2.3.2. Covalent immobilization strategy. Thiolated-probe solutions, $0.25 \mu \mathrm{L}$ in $\mathrm{PBS}$ at the desired concentration, were spotted onto vinyl-functionalized planar SOI chips with a non-contact dispenser in a microarray format including 4 replicas of each spot in all experiments. The microarray formed was exposed to UV light (50 $\mathrm{mW} \cdot \mathrm{cm}^{-2}, 254 \mathrm{~nm}$ ) for $30 \mathrm{~s}$ to induce photoimmobilization. After exposure, the chips were washed with PBST, water and dried with air. Then, the fluorescence of the Cy5-labeled probes was measured.

$10 \mu \mathrm{L}$ of a PBS $10 \mu \mathrm{M}$ solution of MB 2 was dropped over the vinyl-functionalized DPI chip. The chip was then covered with a quartz coverslip and exposed to UV light $\left(50 \mathrm{~mW} \cdot \mathrm{cm}^{-2}, 254 \mathrm{~nm}\right)$ for $30 \mathrm{~s}$ to induce photoimmobilization. After exposure, the chips were washed with PBST, water and dried with air. 
2.3.3. Protein recognition assay. A drop of a solution containing streptavidin $\left(10 \mu \mathrm{L}, 50 \mu \mathrm{g} \cdot \mathrm{mL}^{-1}\right.$ in PBST) was drop-casted over chips with immobilized MBs incorporating biotin. It was then extended over the entire surface with a coverslip and incubated at room temperature for $30 \mathrm{~min}$. Then, after removing the coverslip, the chips were washed with PBST, water and air dried.

2.3.4. Hybridization strategy. $10 \mu \mathrm{L}$ of $0.5 \mu \mathrm{M}$ solutions of the target oligonucleotide in SSC buffer were spotted over the microarrays and extended homogeneously over the entire chip surface with a coverslip. The chips were incubated at $37^{\circ} \mathrm{C}$ for $1 \mathrm{~h}$. Then, the chips were washed with diluted SSC buffer and distilled water. Finally, the fluorescence of the formed microarray was measured.

All protein recognitions and hybridizations were performed in duplicate to test the reproducibility of each experiment and only one of the chips is shown in the images for clarity reasons.

\section{RESULTS AND DISCUSSION}

\subsection{Immobilization of MB probes.}

For the development of the microchips, MBs were covalently attached to the surface of the chip. In this work, immobilization of MBs was performed using optimized thiol-ene chemistry (TEC) that was previously developed in our group for linear oligonucleotides with a few modifications. [11,14] TEC provides robust and controlled anchoring of biomolecules by using UV-light, in a fast, straightforward and biocompatible manner. Fluorescence labeled MB 1 was used to determine the immobilization yield by interpolation in the previously constructed calibration curve (Fig. S1a and S1b). Growing concentrations of MB 1 were spotted with the noncontact automatic dispenser (from 1 to $10 \mu \mathrm{M}$ ) and immobilized with UV-light as previously described. The fluorescence was recorded after irradiation to construct the calibration curve. Then, the chips were washed and the registered fluorescence values interpolated in the corresponding calibration curve. Only $30 \mathrm{~s}$ irradiation time was necessary, achieving maximum immobilization densities of up to $14.5 \pm 0.5 \mathrm{pmol} \cdot \mathrm{cm}^{-2}$ (Fig. S1d). This value compares very well with previously reported immobilization yields of thiolated probes attached by TEC, where maximal immobilization densities were around $6 \mathrm{pmol} \cdot \mathrm{cm}^{-2} \cdot[14,11]$ The use of a high power lamp $\left(50 \mathrm{~mW} / \mathrm{cm}^{2}\right)$ permitted to drastically reduce irradiation times, and thus possible degradations of the surface and/or fluorophores also decreased giving rise to higher immobilization densities.

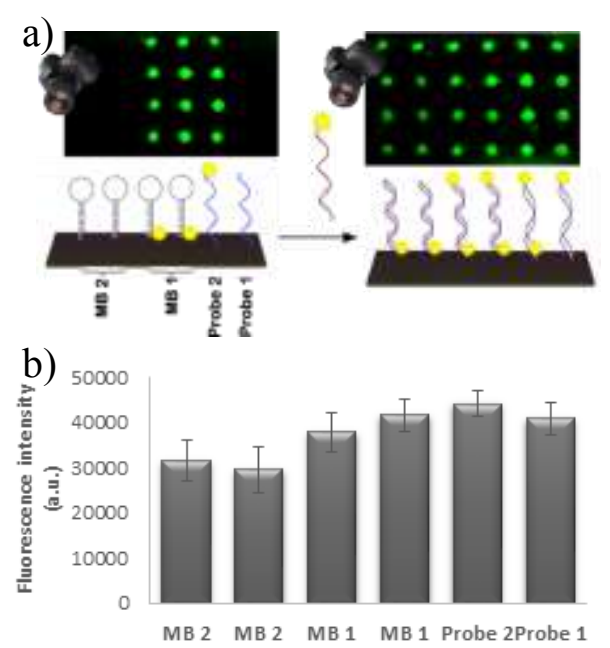

Fig. 1. a) Scheme of the microarray used to test the MB anchoring and hybridization. b) Fluorescence quantification after hybridization.

\subsection{Hybridization assays.}

For the hybridization of target probes, comparison between linear oligonucleotides and MB performances for hybridization with their complementary strands were interrogated on planar SOI surfaces. Microarrays were fabricated using thiolated MB 1 and MB 2 (10 and $20 \mu \mathrm{M}$ concentrations each) and linear oligonucleotides Probe 1 and Probe $2(10 \mu \mathrm{M})$ over vinylfunctionalized-SOI planar chips. After immobilization, the fluorescence of the microarray was registered with the SFR, and then the microarrays were hybridized with fluorescent Target 1 $(0.5 \mu \mathrm{M}$ in SSC $1 \mathrm{x})$ for $1 \mathrm{~h}$ at $37^{\circ} \mathrm{C}$. After washing, the fluorescence was registered again as shown in Fig. 1.

Very good performances that are comparable with that of linear oligonucleotides were obtained for all probes, as seen on fig. $1 \mathrm{~b}$. The suitability of the method for hybridization assays was tested by immobilizing increasing concentrations of unlabeled thiolated MB 2 to the vinyl functionalized SOI surfaces. Then, the fluorescence labeled complementary strand at decreasing concentrations was hybridized at $37^{\circ} \mathrm{C}$ for $1 \mathrm{~h}$. The slides were

finally washed and fluorescence measured (Fig. S1c). The lowest concentration of target that we were able to detect experimentally was $12.5 \mathrm{nM}$, which is in the order of the estimated limit of detection for the target and the detection device used for these experiments. Such estimated LOD was calculated as the blank signal, plus 3 times its standard deviation, and resulted $9.9 \pm 1.1 \mathrm{nM}$. These results indicate that the method presents high sensitivity and high hybridization densities having good correlation with the amount of immobilized probe. Thus, it was demonstrated 
that MBs can be covalently attached to functionalized surfaces by TEC and they are bioavailable for further hybridization with the complementary strand.

\subsection{Multiplexing and protein recognition capabilities.}

The selectivity of the approach was evaluated through hybridization with different targets. A set of different biotinylated MB 2, MB 4, not biotinylated MB 3, linear oligonucleotides Probe 1 and Probe 2, MB 1 and biotinPEG-SH as control probes, were immobilized on several planar SOI chips by TEC chemistry at different concentrations (Fig. 2a). Before hybridization, chips only show fluorescence for MB 1 and Probe 2, demonstrating covalent immobilization. Then, sets of two chips were hybridized with Target 1,2 and 3 complementary to MB 2, 3 and 4, respectively. After hybridization, fluorescence was observed only where full complementarity was present, proving selectivity of the MBs in microarray format (Fig. 2 b and Fig. S2 for fluorescence intensity values).

Also, the ability of biotinylated MBs to recognize different streptavidin proteins on a selective manner was tested. The same microarray was revealed with the corresponding streptavidin solution as previously described in the experimental section. Then, fluorescence was measured for Cy5-streptavidin and colorimetric development was observed after development with tetramethylbenzidine (TMB) for the Horseradish Peroxidase (HRP) labeled streptavidin (Fig. 2c). In both cases, positive results were observed only on the spots of the microarray where biotinlabeled MBs were present (MB 1, 2 and 4), as well as on the positive control with biotin-PEG-SH, proving high selectivity and sensitivity for the biotin-streptavidin interaction. When Cy5 labeled streptavidin was used, fluorescence was also observed for MB 1 and Probe 2 as were also labeled with Cy5, but in the case of HRPstreptavidin, Probe 2 did not produced a positive result whereas MB 1 appeared positive as it has biotin as well as Cy5.

a)

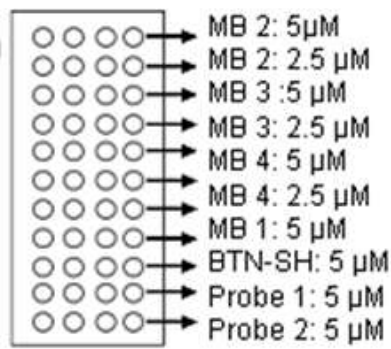

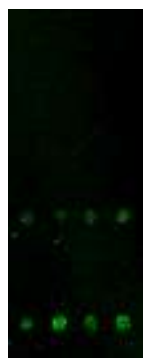

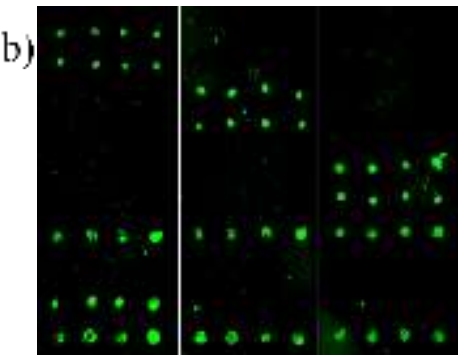

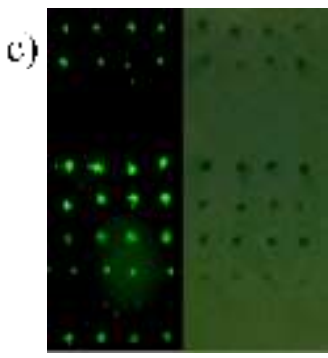

Fig. 2. a) Microarray printed with a non-contact dispenser and fluorescence observed after photoimmobilization of probes as measured with the SFR. b) Fluorescence measured with the SFR after hybridization with each fluorescence labeled target (from left to right: Target 1,2 and 3). c) Chips revealed with Cy5 streptavidin (left) and Horseradish Peroxidase (HRP) streptavidin (right, after TMB processing). Fluorescence intensities after hybridizations are included in Fig. S2)

Finally, to employ this approach for the construction of biosensors based on different reporters incorporated through biotin-streptavidin interactions, the MB probes need to be accessible for hybridization after the protein is incorporated to the MB. This process was tested using chips where several MBs were immobilized both with and without biotin labeling (fig. S3). The chips were revealed with streptavidin with Cy5 and HRP labeling to prove the incorporation of streptavidin. After streptavidin was added to the MBs, the chips with MBs and HRP-streptavidin were hybridized with Target 1 (Cy5 labeled) and measured with the SFR. Fluorescence was observed selectively for full complementary probes although the fluorescence intensity was $36.7 \pm 1.3 \%$ lower than that observed when unlabeled streptavidin was not attached to the MB. Finally, color development by TMB substrate was interrogated on the hybridized chips, proving the presence of HRP-streptavidin as the blue precipitate appears selectively only on biotin-labeled MBs.

Therefore, working in microarray, MBs are able to specifically hybridize after streptavidin-biotin binding. However, when using streptavidin-HRP the hybridization yield decreases by $37.9 \pm 1.7 \%$ compared to that observed for streptavidin-free MBs, although the streptavidin-biotin recognition remains specific as shown when the chips are developed with TMB after streptavidin-HRP recognition and hybridization with the target.

\subsection{DPI analysis.}


DPI provides a record of the phase changes in the Transverse Electrical (TE) and Transverse Magnetic (TM) polarizations of light through the waveguide in real time. The variations in TM and TE modes are transformed in variations of thickness, mass and density of the monolayer, giving information of the conformational changes that occur when the immobilized MB recognizes streptavidin or hybridizes with the corresponding complementary strand. Silicon oxinitride chips were functionalized with vinyltriethoxysilane by CVD, as previously reported, and thiolated MB $2(10 \mu \mathrm{M})$ was attached over the entire surface of the chip using TEC chemistry.[19,17] Different solutions were injected on each channel and all events are recorded in real-time (Fig. 3). Then, all discrete data that resulted from the experiment are summarized in table 2.

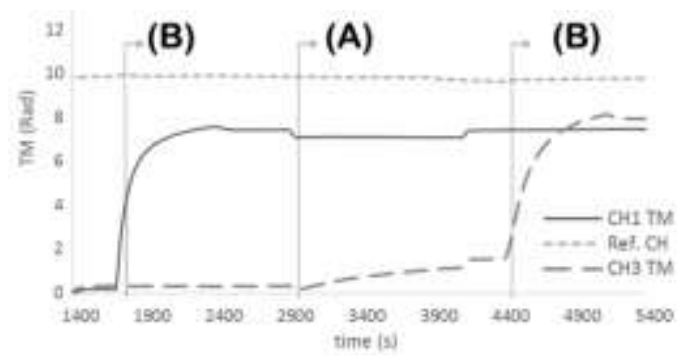

Fig. 3. DPI experiment where the streptavidin injection (a) at $\mathrm{t}=1500 \mathrm{~s}(\mathrm{CH} 1)$ and (c) $5500 \mathrm{~s}(\mathrm{CH} 3)$ and Target 1 injection (b) at $\mathrm{t}=3000 \mathrm{~s}(\mathrm{CH} 1$ and 3) can be observed as they produce changes in the TE and TM polarizations of light.

In channel 1, two injections were performed to study the hybridization after protein addition. First streptavidin was flown followed by injection of Target 1 . At the beginning of the experiment, the MB is attached to the surface and a mass increase of $1.32 \mathrm{ng} \cdot \mathrm{mm}^{-2}$ is observed after streptavidin injection. This $1.32 \mathrm{ng} \cdot \mathrm{mm}^{-2}$ represents $2.49 \mathrm{pmol} \cdot \mathrm{cm}^{-2}$ of streptavidin on the surface, which is equivalent to $80 \%$ of a close packed monolayer of streptavidin (calculated considering the protein a $6 \mathrm{~nm}$ diameter sphere, $3.11 \mathrm{pmol} \cdot \mathrm{cm}^{-2}$ ). From the microarray studies, the probe density when a $10 \mu \mathrm{M}$ concentration of MB was attached to the surface by TEC chemistry was $14.5 \pm 0.5 \mathrm{pmol} \cdot \mathrm{cm}^{-2}$. Therefore, this amount of streptavidin, which is nearly the maximum allowed on the surface, only represents $18 \%$ of the biotinylated MB to be bonded to streptavidin.

Regarding the thickness increase, a $3.2 \mathrm{~nm}$ layer thickness increase is observed after streptavidin binding. This can be explained considering that streptavidin binds to the $\mathrm{MB}$ at the surface in a stand-up mode, providing its longer side to be quasi-orthogonal to the surface, and thus, overpassing the high defined by the MB layer (3-4 nm) by approximately $3 \mathrm{~nm}$ as represented in Fig. 4 . When hybridization takes place after the addition of streptavidin (Fig. 4), the amount of mass loading on the surface $\left(0.323 \mathrm{ng} \cdot \mathrm{mm}^{-2}\right)$ corresponds to $4.9 \mathrm{pmol} \cdot \mathrm{cm}^{-2}, 33 \%$ hybridization efficiency, which correlates very well with the previously observed hybridization yield on planar surfaces for MBs incorporating streptavidin. Finally, analyzing the thickness increase after hybridization $(0.8 \mathrm{~nm})$ it can be interpreted that, after the hybridization, the MB opens the hairpin structure and elongates (theoretical length of a dsDNA having $25 \mathrm{bp}$ is $8.5 \mathrm{~nm}$ ), which is also consistent with the slight decrease observed in density.

In channel 3, two injections were performed to study hybridization to MBs, first, Target 1 was flown and then, streptavidin was injected to the hybridized system. In this case, when hybridization takes place before streptavidin binding, the mass loading on the surface results $0.776 \mathrm{ng} \cdot \mathrm{mm}^{-2}$. Considering the target's molecular weight $\left(6486 \mathrm{~g} \cdot \mathrm{mol}^{-1}\right)$, the mass loading corresponds to $11.96 \mathrm{pmol} \cdot \mathrm{cm}^{-2}$ of target hybridized on the surface, which means $85 \%$ hybridization efficiency for the immobilized MB $\left(14.5 \pm 0.5 \mathrm{pmol} \cdot \mathrm{cm}^{-2}\right)$.

Table 2. DPI analysis of the monolayer upon streptavidin/probe injections. 


\begin{tabular}{|c|c|c|c|c|c|c|c|}
\hline Injections $\mathrm{CH} 1$ & RI & $\begin{array}{l}\text { Thickness } \\
(\mathrm{nm})\end{array}$ & $\begin{array}{l}\text { Mass } \\
\left(\mathrm{ng} \cdot \mathrm{mm}^{-2}\right)\end{array}$ & $\begin{array}{l}\text { Density } \\
\left(\mathrm{g} \cdot \mathrm{cm}^{-3}\right)\end{array}$ & $\begin{array}{l}\Delta \text { Thickness } \\
(\mathrm{nm})\end{array}$ & $\begin{array}{l}\Delta \text { Mass } \\
\left(\mathrm{ng} \cdot \mathrm{mm}^{-2}\right)\end{array}$ & $\begin{array}{l}\Delta \text { Density } \\
\left(\mathrm{g} \cdot \mathrm{cm}^{-3}\right)\end{array}$ \\
\hline raw chip & 0 & 0 & 0 & 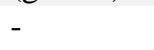 & & & \\
\hline Streptavidin & $\begin{array}{l}1.4181 \\
( \pm 0.0001)\end{array}$ & $\begin{array}{l}3.20 \\
( \pm 0.01)\end{array}$ & $\begin{array}{l}1.3191 \\
( \pm 0.0001)\end{array}$ & $\begin{array}{l}0.4118 \\
( \pm 0.0001)\end{array}$ & $\begin{array}{l}3.20 \\
( \pm 0.01)\end{array}$ & $\begin{array}{l}1.3191 \\
( \pm 0.0001)\end{array}$ & $\begin{array}{l}0.4118 \\
( \pm 0.0001)\end{array}$ \\
\hline Target 1 & $\begin{array}{l}1.4175 \\
( \pm 0.0001)\end{array}$ & $\begin{array}{l}4.01 \\
( \pm 0.01)\end{array}$ & $\begin{array}{l}1.6421 \\
( \pm 0.0001)\end{array}$ & $\begin{array}{l}0.4086 \\
( \pm 0.0001)\end{array}$ & $\begin{array}{l}0.81 \\
( \pm 0.01)\end{array}$ & $\begin{array}{l}0.3229 \\
( \pm 0.0001)\end{array}$ & $\begin{array}{l}-0.0031 \\
( \pm 0.0001)\end{array}$ \\
\hline Injections $\mathrm{CH} 3$ & RI & $\begin{array}{l}\text { Thickness } \\
(\mathrm{nm})\end{array}$ & $\begin{array}{l}\text { Mass } \\
\left(\mathrm{ng} \cdot \mathrm{mm}^{-2}\right)\end{array}$ & $\begin{array}{l}\text { Density } \\
\left(\mathrm{g} \cdot \mathrm{cm}^{-3}\right)\end{array}$ & $\begin{array}{l}\Delta \text { Thickness } \\
(\mathrm{nm})\end{array}$ & $\begin{array}{l}\Delta \text { Mass } \\
\left(\mathrm{ng} \cdot \mathrm{mm}^{-2}\right)\end{array}$ & $\begin{array}{l}\Delta \text { Density } \\
\left(\mathrm{g} \cdot \mathrm{cm}^{-3}\right)\end{array}$ \\
\hline raw chip & 0 & 0 & 0 & - & & & \\
\hline Target 1 & $\begin{array}{l}1.3867 \\
( \pm 0.0001)\end{array}$ & $\begin{array}{l}3.26 \\
( \pm 0.01)\end{array}$ & $\begin{array}{l}0.7761 \\
( \pm 0.0001)\end{array}$ & $\begin{array}{l}0.2379 \\
( \pm 0.0001)\end{array}$ & $\begin{array}{l}3.26 \\
( \pm 0.01)\end{array}$ & $\begin{array}{l}0.7761 \\
( \pm 0.0001)\end{array}$ & $\begin{array}{l}0.2379 \\
( \pm 0.0001)\end{array}$ \\
\hline streptavidin & $\begin{array}{l}1.3819 \\
( \pm 0.0001)\end{array}$ & $\begin{array}{l}4.34 \\
( \pm 0.01)\end{array}$ & $\begin{array}{l}0.9206 \\
( \pm 0.0001)\end{array}$ & $\begin{array}{l}0.2119 \\
( \pm 0.0001)\end{array}$ & $\begin{array}{l}1.08 \\
( \pm 0.01)\end{array}$ & $\begin{array}{l}0.1444 \\
( \pm 0.0001)\end{array}$ & $\begin{array}{l}-0.0259 \\
( \pm 0.0001)\end{array}$ \\
\hline
\end{tabular}

The theoretical density of dsDNA is $1.7 \mathrm{~g} \cdot \mathrm{cm}^{-3}$, and considering that the theoretical coverage of a closed-packed monolayer of dsDNA is $45-50 \mathrm{pmol} \cdot \mathrm{cm}^{-2},[20]$ the previously calculated surface density of hybridized target on the monolayer of $12 \mathrm{pmol} \cdot \mathrm{cm}^{-2}$ would result in $0.450 \mathrm{~g} \cdot \mathrm{cm}^{-3}$, as far as half of the mass was already on the surface before hybridization, a density increase of $0.225 \mathrm{~g} \cdot \mathrm{cm}^{-3}$ should be expected, which fits the experimental data $\left(0.238 \mathrm{~g} \cdot \mathrm{cm}^{-3}\right)$.

The length of a dsDNA of $25 \mathrm{bp}$ is $8.5 \mathrm{~nm}$ and as a thickness increase of $3.26 \mathrm{~nm}$ was observed, a tilt angle in the dsDNA layer of $53^{\circ}$ is estimated (similar tilt angle values have been previously reported in the literature).[21]

After the hybridization step, streptavidin is flown over the surface obtaining a mass loading of $0.144 \mathrm{ng} \cdot \mathrm{mm}^{-2}$. This corresponds to $0.27 \mathrm{pmol} \cdot \mathrm{cm}^{-2}$ of bonded streptavidin, which represents $9 \%$ of the protein close-packed monolayer. In this low-coverage conditions, the thickness given by the instrument is an averaged value from the thickness of the monolayer areas with and without the protein. $[22,23]$ Then, only $0.36-0.54 \mathrm{~nm}$ of thickness increase are possible due to the streptavidin layer. However, the thickness increase observed in this step is $1.08 \mathrm{~nm}$. This can be interpreted considering that the streptavidin binding forces the dsDNA to stand up slightly more orthogonal to the surface, being this conformational change in the dsDNA the main responsible of the thickness increase. Thus, from the thickness increase, a tilt angle of $67^{\circ}$ is calculated, which is $14^{\circ}$ higher than that obtained after hybridization. This interpretation also fits with the fact that the density is almost unaltered, and slightly decreases, because the layer thickness is higher but without significantly increasing the mass. 
After the experiment with DPI was finished, as Target 1 was labeled with Cy5, the chip fluorescence was registered in the surface fluorescence reader (Fig. S4), showing high fluorescence on both channels, which confirms that hybridization was successful in both of them.

\section{CHANNEL 1 CHANNEL 3}
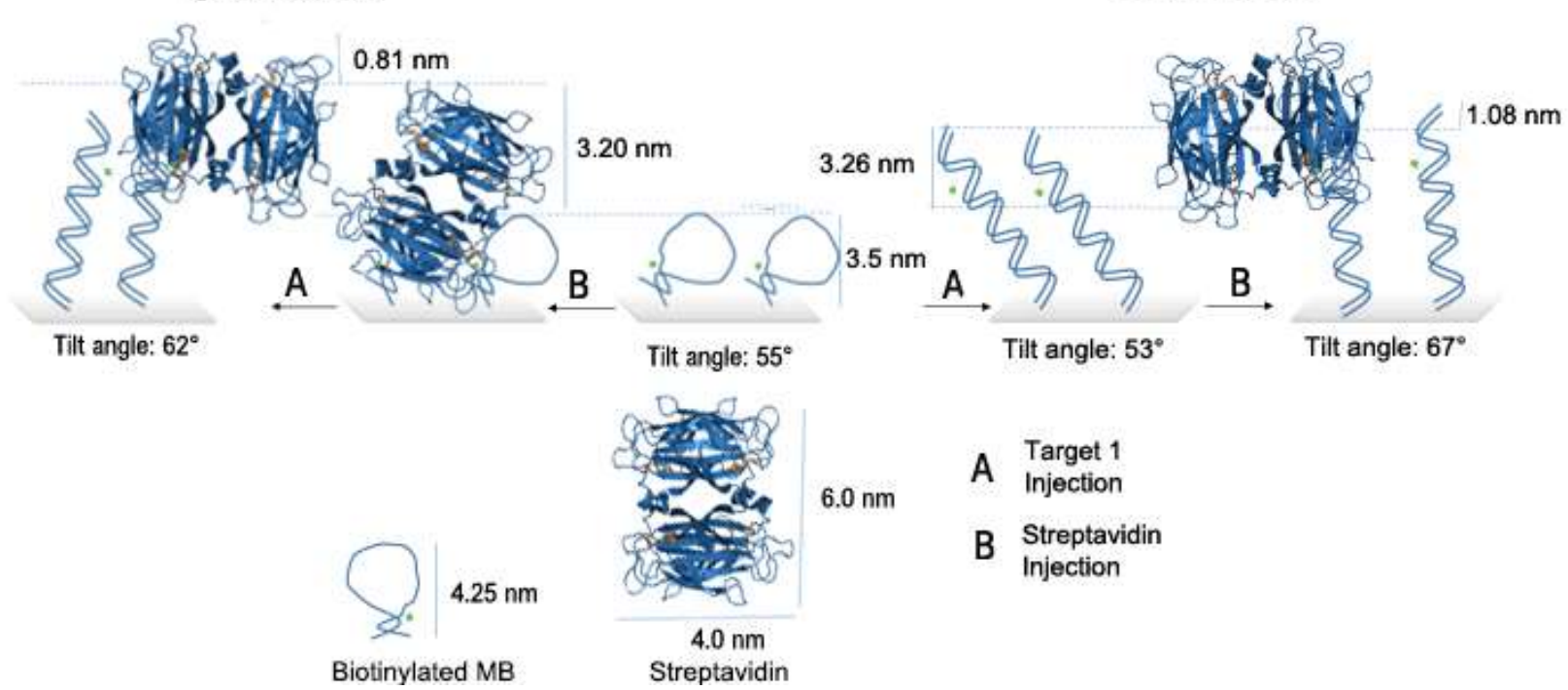
A Target 1
Injection
B Streptavidin
Injection

Fig. 4. Representation of the MB proposed conformational changes that occur when Target 1 or streptavidin are flown over channel 3 (left) or channel 1 (right). Note that the same final state was observed in both channels with similar thickness, mass and density variations.

\section{CONCLUSIONS}

During these studies, immobilization of MBs was achieved on planar surfaces with only 30 s photoimmobilization, producing high immobilization densities $\left(14.5 \pm 0.5 \mathrm{pmol} \cdot \mathrm{cm}^{-2}\right)$. The anchored MBs were able to hybridize with the complementary target with high selectivity and sensitivity in microarray format. The estimated limit of detection, obtained for the methodology in microarray format, was $9.9 \pm 1.1 \mathrm{nM}$ which compares very well with previous methodologies developed on planar surfaces. MBs with a biotin moiety in one side of the stem were able to incorporate a streptavidin protein in a straightforward manner by strong biotin-streptavidin interaction. This process opens the possibility to incorporate a wide range of streptavidin-linked reporters that are currently commercially available. MBs with the attached streptavidin were able to hybridize with the complementary target, although the hybridization yield slightly diminishes in the presence of the protein.

From the DPI analysis it was concluded that the hybridization with the target, when a protein is linked to the MB, promotes an effective distancing of the protein away from the surface as deduced from the variations in mass, density and thickness. The final relative position of the dsDNA with an attached protein is tilted $62-67^{\circ}$ to the surface, even higher angle than that obtained for protein-free dsDNA $\left(52^{\circ}\right)$. The high distancing of the reporter from the surface upon hybridization can be directly applied to novel label-free biosensors based on MB technology as virtually any reporter can be incorporated to MBs without significantly affecting the hybridization process. Therefore, attaching a high refractive index nanoparticle to MBs can amplify the optical change produced by an interferometric biosensor, or the incorporation of a metallic nanoparticle can be directly applied in surface plasmon resonance or electrochemical sensors.

\section{Acknowledgements}

This research was funded by the European Union Horizon 2020 program (ICT-644242 SAPHELY project), the MINECO projects CTQ/2013/45875-R, and CTQ/2016/75749-R, FEDER, and GVA PROMETEO II 2014/40.

\section{REFERENCES}


1. Huertas CS, Fariña D, Lechuga LM (2016) Direct and Label-Free Quantification of Micro-RNA-181a at Attomolar Level in Complex Media Using a Nanophotonic Biosensor. ACS Sensors 1 (6):748-756.

2. Bañuls M-J, González-Pedro V, Barrios CA, Puchades R, Maquieira Á (2010) Selective chemical modification of silicon nitride/silicon oxide nanostructures to develop label-free biosensors. Biosens Bioelectron 25 (6):14601466.

3. Zhang G-J, Chua JH, Chee R-E, Agarwal A, Wong SM (2009) Label-free direct detection of MiRNAs with silicon nanowire biosensors. Biosens Bioelectron 24 (8):2504-2508.

4. Sassolas A, Leca-Bouvier BD, Blum LJ (2008) DNA Biosensors and Microarrays. Chem Rev 108 (1):109-139.

5. Chen T, Tan W (2013) Molecular Beacons on Solid Surfaces. In: Yang CJ, Tan W (eds) Molecular Beacons. Springer Berlin Heidelberg, Berlin, Heidelberg, pp 75-90.

6. Wang K, Tang Z, Yang CJ, Kim Y, Fang X, Li W, Wu Y, Medley CD, Cao Z, Li J, Colon P, Lin H, Tan W (2009) Molecular Engineering of DNA: Molecular Beacons. Angew Chem Int Ed 48 (5):856-870.

7. Zheng J, Yang R, Shi M, Wu C, Fang X, Li Y, Li J, Tan W (2015) Rationally designed molecular beacons for bioanalytical and biomedical applications. Chem Soc Rev 44 (10):3036-3055.

8. Guo Q, Bai Z, Liu Y, Sun Q (2016) A molecular beacon microarray based on a quantum dot label for detecting single nucleotide polymorphisms. Biosens Bioelectron 77:107-110.

9. Mei Z, Tang L (2016) Surface plasmon coupled fluorescence enhancement based on ordered gold nanorod array biochip for ultra-sensitive DNA analysis. Anal Chem 89 (1):633-639.

10. Li S, Wang Y, Gao C, Ge S, Yu J, Yan M (2015) "Signal-off” photoelectrochemical DNA sensing strategy based on target dependent DNA probe conformational conversion using CdS quantum dots sensitized TiO2 nanorods array as photoactive material. J Electroanal Chem 759:38-45.

11. Escorihuela J, Bañuls M-J, Grijalvo S, Eritja R, Puchades R, Maquieira Á (2014) Direct Covalent Attachment of DNA Microarrays by Rapid Thiol-Ene "Click" Chemistry. Bioconjugate Chem 25 (3):618-627.

12. Yao G, Tan W (2004) Molecular-beacon-based array for sensitive DNA analysis. Anal Biochem 331 (2):216223.

13. Du H, Strohsahl CM, Camera J, Miller BL, Krauss TD (2005) Sensitivity and Specificity of Metal SurfaceImmobilized "Molecular Beacon" Biosensors. J Am Chem Soc 127 (21):7932-7940.

14. Escorihuela J, Banuls MJ, Puchades R, Maquieira A (2012) DNA microarrays on silicon surfaces through thiolene chemistry. Chem Commun 48 (15):2116-2118.

15. Zhao W-W, Wang J, Xu J-J, Chen H-Y (2011) Energy transfer between CdS quantum dots and Au nanoparticles in photoelectrochemical detection. Chem Commun 47 (39):10990-10992.

16. Bayer EA, Wilchek M (1990) [4] Biotin-binding proteins: Overview and prospects. In: Meir W, Edward AB (eds) Methods in Enzymology, vol 184. pp 49-51.

17. Escorihuela J, González-Martínez MÁ, López-Paz JL, Puchades R, Maquieira Á, Gimenez-Romero D (2015) Dual-Polarization Interferometry: A Novel Technique To Light up the Nanomolecular World. Chem Rev 115 (1):265-294.

18. Mira D, Llorente R, Morais S, Puchades R, Maquieira A, Marti J High-throughput screening of surfaceenhanced fluorescence on industrial standard digital recording media. In: Proc. SPIE, 2004. pp 364-373

19. Volcke C, Gandhiraman RP, Gubala V, Doyle C, Fonder G, Thiry PA, Cafolla AA, James B, Williams DE (2010) Plasma functionalization of AFM tips for measurement of chemical interactions. J Colloid Interface Sci 348 (2):322-328.

20. Georgiadis R, Peterlinz KP, Peterson AW (2000) Quantitative Measurements and Modeling of Kinetics in Nucleic Acid Monolayer Films Using SPR Spectroscopy. J Am Chem Soc 122 (13):3166-3173.

21. Elhadj S, Singh G, Saraf RF (2004) Optical Properties of an Immobilized DNA Monolayer from 255 to $700 \mathrm{~nm}$. Langmuir 20 (13):5539-5543.

22. Xu K, Ouberai MM, Welland ME (2013) A comprehensive study of lysozyme adsorption using dual polarization interferometry and quartz crystal microbalance with dissipation. Biomaterials 34 (5):1461-1470.

23. Cowsill BJ, Coffey PD, Yaseen M, Waigh TA, Freeman NJ, Lu JR (2011) Measurement of the thickness of ultra-thin adsorbed globular protein layers with dual-polarisation interferometry: a comparison with neutron reflectivity. Soft Matter 7 (16):7223-7230. 